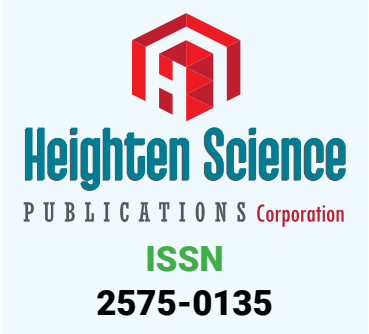

*Address for Correspondence: Dr. Aboul-Ata $\mathrm{E}$ Aboul-Ata, Department of Genetic Engineering Res, Egyptian Company for Blood, Egypt Transfusion, VACSERA, Dokki, Giza, Egypt, Email: aboulata_nady@hotmail.com; aeaboulata@gmail.com

Submitted: 08 July 2017

Approved: 08 August 2017

Published: 09 August 2017

Copyright: @ 2017 Janahi EM, et al. This is an open access article distributed under the Creative Commons Attribution License, which permits unrestricted use, distribution, and reproduction in any medium, provided the original work is properly cited.

\section{HBV: Genomic Structure, HBVsAg Isolation and innovative Virotherapy Initiation in the Middle East}

\author{
Essam M Janahi ${ }^{1}$, Aboul-Ata E Aboul-Ata ${ }^{2 *}$, I M El-Kalamawy ${ }^{3}$, \\ Kathleen Hefferon ${ }^{4}$ and Amal Mahmoud ${ }^{5}$ \\ 'Department of Biology, College of Science, University of Bahrain, P.O. Box 32038, Sakhir, \\ Kingdom of Bahrain, Egypt \\ 2Department of Genetic Engineering Res, Egyptian Company for Blood, Egypt Transfusion, \\ VACSERA, Dokki, Giza, Egypt \\ ${ }^{3}$ Department of Molecular Biology, Cornell University, Ithaca, NY, USA \\ ${ }^{4}$ Genetic Engineering Inst, Sadat University, Sadat City, Munofia, Egypt \\ ${ }^{5}$ Molecular Biology Lab. II, Plant Virus and Phytoplasma Res. Sec, Plant Path. Res. Inst, ARC, \\ POB 12619, Giza, Egypt
}

\section{BRIEF INFORMATION}

Hepatitis B virus (HBV) is one of the world's major infectious diseases with 350 million people who are chronic carriers of HBV [1]. Significant minorities go on to develop liver cirrhosis or hepatocellular carcinoma and over 1 million die annually from HBV-diseased liver. Janahi E. at faculty of science, Bahrain University, Bahrain has submitted the following information [2], on HBV-genome organization as part of his Ph.D. degree (2007) in Imperial College, England. HBV genomic organization has 4 Open Reading Frames (ORFs) i.e. Pre-S/S Gene, Pre-C/C ORF, P ORF and X ORF. Regulatory Elements has 4 promoters (pre S2, pre S1, C promoters and X promoters), Pregenomic RNA, Enhancers (Enh 1 and Enh 2) where they are involved in cccDNA formation, Glococorticoid-Responsive Element which is located in X ORF and P ORF overlapping, Polyadenylation Signal (Direct Repeat 1 (DR1) and Direct Repeat 2 (DR2)), EpsilonStem Loop and Post-Transcriptional Regulatory Element. HBV genotype D is prevalent in our Middle East area. The HBV genome is a partially relaxed-circular dsDNA molecule consisting of a full length strand (minus strand) with a single unique nick and a complementary (positive strand) of variable length. HBV is considered as a pararetrovirus because its replication involves the reverse transcription of an intermediateRNA function, of pre-genomic RNA (pgRNA). Replication of HBV genome starts with the encapsidation of the pgRNA and encodes HBV polymerase into an immature nucleocapsid formed by the viral core antigen. Inside the immature nucleocapsid, the viral polymerase converts pgRNA into minus-strand DNA, which in turn is used as a template for the synthesis of the plus-strand DNA, resulting in the formation of the characteristic mature double-stranded, relaxed circular DNA molecule [2]. HBVsAg has been isolated from Egyptian samples and identified using RTPCR [3-5]. Polymerase and HBVsAg regions have been also isolated and identified [5]. HBVsAg (S) gene has been identified at the band size $25.42 \mathrm{kDa}[3,4]$. Virotherapy for plant-based vaccine structure has been speculated for future work. Proposed CMV-HBVsAg chimeric-virus construct. Cucumber mosaic virus (CMV) $26 \mathrm{kDa}$ hybrid coat protein (CP D/S) gene for 2 strains (CMV/S and CMV/D) were isolated and amplified from sgRNA 4 using $\mathrm{F}$ and $\mathrm{R}$ primers. Replicase gene (RP) and $30 \mathrm{kDa}$ movement protein gene (MP) were used. 
Promoter (35sP). Nopaline synthase terminator (Nos3T) are constructed between Right and left boarder (RB and LB). Proposed BeYDV-HBVsAg chimeric virus construct. HBVsAg (S) protein -Bean Yellow Dwarf Begomovirus chimeric virus [6]. Long and short intergenic regions (LIR \& SIR) of bean yellow dwarf Gemini virus as well as capsid protein (CP), movement protein (MP) and replication-associated protein (RepA) genes and as well as 35s P and Nos3T will be constructed between Right and left boarder (RB and LB). Brief processing of vaccine production is as follow: a. Virus and RNA sources, which is HBVsAg that has isolated as two isolates from Egypt by El-Kalamawy et al. [4]. Also Mahmoud and Hashem [5], have isolated HBVsAg and HBVpAg. Also, Elghannam et al. [3], have isolated HBVsSg. b. Computer-Assisted Analysis, c. in vitro Construction of Chimeric Viruses (1. Construct Design, 2. Target Gene (Sub-units), Gene Promotors, Terminators and Orientation, 3. Virus-Based Vector (Recombinant Viral Genes), 4. CP, Replicase and Movement Protein Genes, 5. Chimeric Virus Stability. 6. Plant Bioreactor Inoculation, Vacuum and Agrobacterium infiltration, 7. Transient Expression, 8. Gene Transformation, 9. Plant-expressed Protein Vaccine Confirmation (Symptomatology, EM and WB). Scaling up vaccine-containing plants, Expressed-protein vaccine purification and dosing. Bioreactor host plant has to be chosen.

\section{Proposed Chimeric-Virus Constructs}

Figure 1: Proposed BeYDV-HBVsAg chimeric virus construct. $25.42 \mathrm{kDa}$ HBVsAg (S) protein that was isolated previously (3), will be constructed with Bean Yellow Dwarf Begomovirus for chimeric virus. Long and short intergenic regions (LIR \& SIR) of bean yellow dwarf gemini virus as well as capsid protein (CP), movement protein (MP) and replication-associated protein (RepA) genes and HBVsAg (S) target gene as well as 35s P and Nos3T will be experimented. CaMV 35s promoter (35sP). Nopaline synthase terminator (Nos3T) are constructed between Right and left boarder (RB and LB). Arrow directions are the orientation activities for C1, C2 and V1, V2 with intron (Int.) The construct will be inserted in pBY002 binary vector $\mathrm{H} 5 \alpha$ E. coli cell to be transfected into suitable bioreactors [1]. Kathleeh Hefferon has used Gemini Bean Yellow Dwarf Virus as vector for getting plant-based HBV vaccine transfected in tomatoes and tobacco [5].

Figure 2: $25.42 \mathrm{kDa}$ HBVsAg (S) (5), has been isolated and identified as target gene from Egyptian patient's samples [3]. CMV-HBVsAg (S) chimeric virus construct is postulated for protection initiation. Cucumber mosaic virus (CMV) $26 \mathrm{kDa}$ hybrid coat protein (CP D/S) gene from 2 strains (CMV/S and CMV/D) will be isolated and amplified from sgRNA 4 using specific primers. Replicase gene (RP) and $30 \mathrm{kDa}$ movement protein gene (MP) will be used for target gene replication. CaMV 35s promoter (35sP)

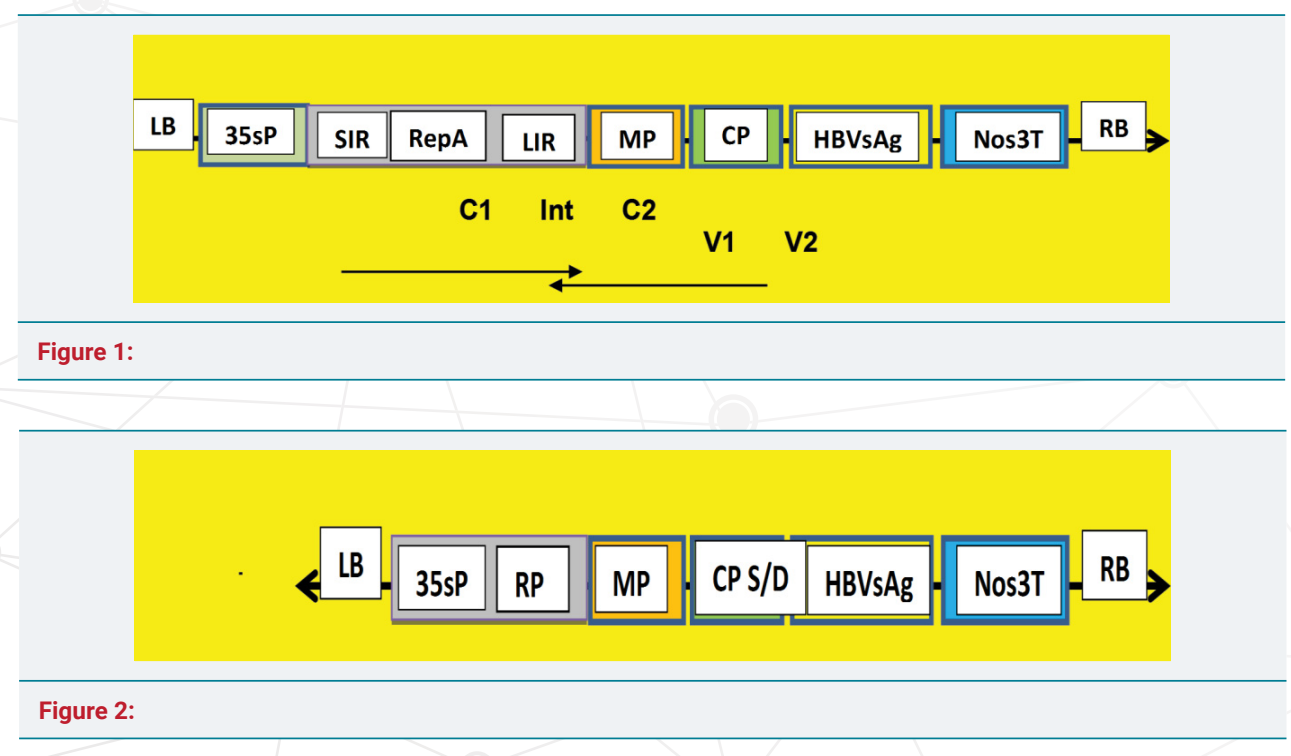


and Nopaline synthase terminator (Nos3T) are also constructed between Right and left boarder (RB and LB). It will be inserted into a binary vector. The construct will be transfected into a bioreactor host [6].

Aboul-Ata et al., have used recombinant CP of Cucumber mosaic virus (CMV) to transfect HCV R9 for chimeric virus into tomato as well as tobacco, lettuce, carrot, cucumber and banana they can be included [1]. Huang et al. have got $0.8 \mathrm{mg} / \mathrm{g}$ of expressed protein when he used HBVcAg. Pniewski et al. [8,9], have got few $\mu \mathrm{g} / \mathrm{g}$ of the protein using HBVsAg and L-and M-HBVsAg.

\section{CONCLUSION}

Expressed recombinant plant protein vaccine is a type of technology that can be a new trend in developing countries to control human and animal viruses i.e. HSV-2, HCV, HBV and others. It includes mass plant production, automated plant transfiction, having enough amount of chimeric virus that having double amount of target gene and the bio-reacted host plant species. All of those previously listed factors are found easily in the $3^{\text {rd }}$ world countries. They also can get in collaboration with developed countries. STD-HBV could be a good tool for vaccination using HBVsAg in those countries. The findings of scientists [7-9], on small amount of expressed protein for the HBV-target genes (HBVsAg, L-and M-HBVsAg, HBVcAg) makes us to speculate another system of plant viral vector construct of chimeric virus.

\section{REFERENCES}

1. Aboul-Ata E Aboul-Ata, Antonella Vitti, Maria Nuzzaci, Ahmad K. El-Attar, Giuseppina 779 Piazzollał et al. Plant-Based Vaccines: Novel and Low-CostPossible Route. For Mediterranean Innovative Vaccination Strategies. Advances in Virus Research. 2014; 89: 1-35.

2. Janahi EM, Hepatitis B virus. thesis. Imperial Collage of Science. 2007.

3. Elghannam MS, Attia AS, Shoeb HA, Hashem AM. Expression and purification of hepatitis B surface antigen S from Escherichia coli; a new simple method. BMC Res Notes. 2012; 5: 125-134. Ref.: https://goo.gl/Wj2VX2

4. El Kalamawy IM, El haddad S, Swelim MA, Hamdy SM, Hanan AF. Molecular variability of S gene of Hepatitis B Virus in Egypt. International J of Virology. 2011; 7: 109-115.

5. Mahmoud Amal, Hashem MH. Bioinformatics analysis of polymerase and envelope genes of hepatitis B virus in Egypt. Egyptian J Virol. 2014; 11: 32-37. Ref.: https://goo.gl/b23S4b

6. Hefferon Kathleen. DNA virus Vectors for Vaccine Production in Plants: Spotlight on Geminiviruses. Vaccines (Basel). 2014; 2: 642-653. Ref.: https://goo.gl/xUzkT2

7. Huang Z, Chen Q, Hjelm B, Arntzen C, Mason H. A DNA replicon system for rapid high-level production of virus-like particles in plants. Biotechnol Bioeng. 2009; 103: 706-714. Ref.: https://goo.gl/sb1JTx

8. Pniewski T, Kapusta J, Bocia g P, Wojciechowicz J, Kostrzak A, et al. Low-dose oral immunization with lyophilized tissue of herbicide-resistant lettuce expressing hepatitis B surface antigen for prototype plant-derived vaccine tablets formulation. J Appl Genet. 2011; 52: 125-136. Ref.: https://goo.gl/pmHbwA

9. Pniewski T, Kapusta J, Bociag P, Kostrzak A, Fedorowicz-Strońska O, et al. Plant expression, lyophilisation and storage of HBV medium and large surface antigens for a prototype oral vaccine formulation. Plant Cell Rep. 2012; 31: 585-595. Ref.: https://goo.gl/WxUgx2 